toujours en faire l'application sans aulre forme de procès : comme on le voil, par les arrếs précilés, la Cour admet aujourd'hui qu'en réalité la loi de isgs a innové, qu'elle a donné aux riverains une propriélé qu’ils ignoraient complètement avant elle, et que la jurisprudence antéricure leur refusail. A partir du jour où la loi a été promulguée, les riverains peuvent revendiquer la propriété du lit, ils sont done soumis à celle loi, aclivement el passivement, pouvant in réclamer le bénélice, à moins que, en le réclamant, ils ne lèsent des droils mis en ceuvre ou déjà cnvisagés par un Iribunal, sous l'empire de la législation ancienne.

Au surplus, la lọi de 1898 semblail elle-même l'indiquer par les mots "sauf titre ou prescription conlraires", qu'elle avail el soin d'insérer dans son texte : "Si les deux rives "apparticnnent à des propriétaires différents, chacun d'cux " a la propriété de la moitić du lit, suivant unc ligne que " l'on suppose tracée au milicu du cours d'eau, sauf. tilr'e "ou prescription contraire".

Du reste, il ne faut pas trop exagérer l'imporlance de celle querclle d'école qui s'est produile au sujet de la loi interprélative ou non : on sait que d'une façon générale, par l'article 2 du Code Civil, la loi ne dispose que pour l'avenir cl n'a pas d'effel rélroactif : mais on admet généralement que cette disposition ne vise pas les lois qui ne feraient que compléter les lois antérieures, el éclaircir des points que celles-ci auraient laissés obscurs. Toulefois, en ce qui concerne Ja loi de 1898 , on ne peul pas dire qu'elle ait fait un éclaircissement d'une situalion indécise, puisqu'en réalité elle a altribué une propriélé vraiment nouvelle, aux riverains des cours d'cau non navigables ni flotlables : et, cûl-elle même ćlé inlerprélalive, que, puisqu'elle devait respecler, d'après son texle, les droils acquis par titres ou par prescription, l'offet n'en aurait guère été modifié.

Disons-le même franchement : on peut très bien supposer que les doux principaux arrêts qui ont proclamé la thèse du caractère interprétatif de la loi auraient pu se dispenser de formuler celte théorie, et qu'ils n'en auraient pas moins été conduits à juger, élant donné les fails, d'une façon absolument identique.

C'est d'ailleurs ce que nous allons examiner.

Dans le premier arrêt, celui de la Cour d'Appel de Grenoble (Delhomme c. Daru, du 3 I octobre $x \delta_{99}$, rapporté dans Dalloz rgoo, deuxième partie, page 350 ), les faits se présenlaient de la façon suivante : sur une parcelle siluée en face des rives dans le lit de la rivière, Delhomme avait placé une canalisation d'cau et un petit pont : les sieurs Daru, qui voulaient continuer à emprunter le lit de la rivière devant Ja rive de Delhomme, pour aller d'un fonds leur appartenant à un autre de leurs fonds, ne se gênèrent pas autrement et supprimèrent simplement la passerelle, si bien qu'à la date du I 2 avril ${ }_{1} 8_{97}$ Delhomme dut agir contre eux : il demanda au Tribunal de le considérer comme propriétaire du lit du ruisseau sur lequel s'exerçail ce passage et assigna les consorts Daru, leur faisant au surplus défense de prali quer tout passage au droit de son fonds.

Sur ces entrefailes, la loi de $x 898$ intervint, et la Cour de Grenoble déclara que celle loi était applicable à la prétention de Delhomme, parce que c'était une loi d'ordre interprélatif ; ct pour prouver ce dernier point elle fit une longue disserlation : il aurait ćtć aussi simple et autrement inallafuable de dire, comme l'a dit la Chambre Civile de la Cour Suprême, dans l'affaire rappclée au début de cet arlicle (Joybert e. Iaval-Noiset), que la loi de i $\delta_{9} \delta$ devait s'appliquer à lous les procis, même ceux pendants au moment de sa promulgation; qu'en conséquence Delhomme avail paríaitenenl raison de revendiquer la propriélé de la parlie du lit siluce en face de sa rive, el qu'il n'aurail pu être paralysé dans son droit que si les sieurs Daru avaienl pul invoquer an lilre ou une possession contrajes, ou évidemment un obslacle juridique, lel que l'enclave par cxemple. Or, précisément, la Cour de Grenoble avait remarqué en fait que Daru, pour se crécr un droil de passagc, ne pouvail alléguer et n'alléguait aucun litre, ni aucun argument lui permettant d'invocuer un droil acquis relalivement au passage. On voil que la discussion sur le caraclère interprélalif ou non de la loi élait absolument inulile.

Il en cst rigourcusement de même pour l'arrêt de la Cour de Cassation rendu dans l'affaire Ott c. Duplan, à la dale du 16 févier rgo4 (Dalloz rgo4, I, 263). La Cour de Nîmes, pour se dire en droit de juger la réclamation de la dame 014 d'après la loi de $\mathbf{1} 898$, avait cru nécessaire de dire que cello loi n'avail qu'un caraclère interprétalif, et que par conséquent elle était applicable à une situation juridique née avant sa promulgation : la Cour de Cassation la suivit dans cette discussion et en a approuvé la conclusion.

Il aurait élé infiniment plus simple de constater que la loi doit être appliquée dans tout jugement qui est rendu au moment où sa promulgation est une chose faite, et d'aulro part les adversaires de la dame Ott n'avaient aucun titre ni aucun droit acquis pour refuser à celte dame le droit de sc dire proprićlaire d'une partie du lit de la Volane : en effet, dans un acte de partage de 1834 il était rcconnu que l'autcur de la dame Ott était propriétaire d'un terrain riverain do cette rivière, et c'est dans cet élat que ce terrain s'était perpétué dans la famille : le 8 avril $\mathrm{r} 898$, la dame Ott voit arriver une loi qui étend sa propriété jusqu'au milieu d'u lit do la Volane : il n'y avail qu'à appliquer cetle loi, puisque la Cour avait elle-même constalć que les adversaires de la dame Ott, simplement en fail, de leur propre autorité privée, sans aucun titre ni aucune prescription, s'étaient installés sur celte partie de la rivière : le déguerpissement est de règle à l'encontre de tous ccux qui occupent sans titre ni prescription, la propriélé d'autrui, celte propriété ne fût-elle que l'humble lit d'une rivière non navigable ni flottable...

Paul Bougault, Amorat à la Cour d'Appel de Lyon.

\section{LA BAUXITE ET LE FOUR ÉLECTRIQUE}

Nous donnons ci-après la conférence faite en mai dernier à la Société Internationale des Electriciens par notre collaborateur M. Fuusir, professeur à la Faculté des Sciences de Grenoble, conférence annoncée dans notre numéro de septembre dernier à propos du "Problime de la fixation industriclle de l'azole ".

Le four électrique permet de faire subir à une même malière première, la bauxite, des transformations diverses, dont l'élude présente quelque intérèt, au point de vue industriel comme au point de vue scientifique.

La bauxite est un minerai de composition fort variable, constitué essentiellement par de l'alumine hydratéc impure ; les impurelés normales sont l'oxyde forrique, la silice et l'oxyde de titane. Fn France, où les giscments do bauxile sont particulièrement nombreux et abondants, on distingue deux qualités principales de minerai: Ja bauxile rouge, richo en oxyde de fer et pauvre en silice; la bauxite. blanche, pauvre on oxyde de fer et riche en silice. Cette distinclion n'a rien d'absolu. On connaît par cxemple des bauxites blanches qui sont à la fois paurres en oxyde de fer et en silice; 
tels sont cerlains minerais américains, qui se rapprochent d'ailleurs plus de la gibbsite que de la bauxite.

Gorindon artificier. - Indépendamment de la bauxite, alumine hydratée, on rencontre dans la nature le corindon ou alumine anhydrc, que sa grande dureté place immédiatement avant le diamant, au numéro 9 de l'échelle de Mohs, el qui est par conséquent un abrasif éncrgique. Les cristaux transparents de corindon pur sont très rares; ils constituent la famille des diverses pierres précieuses alumineuses. On exploite au contraire des gisements assez abondants de corindon opaque, plus ou moins impur, dont la dureté décroît cn même temps que la lencur en alumine, celle-ci étant en moyenne de 80 à 84 pour roo et les impuretés étant surtout formées d'oxyde de fer et de silice. L'émeri est un corindon très impur, mêlé à des quantités parfois considérables d'oxyde de fer (jusqu'à 5o pour roo) et de silice (jusqu'à ro pour 100); la teneur en alumine de l'émeri ne dépasse guère 60 pour roo et sa dureté, inférieure à celle du corindon, varie de 6 à 8 .

Le corindon et l'émeri ont été et sont encore des abrasifs estimés ; la fabrication, vers $1880-1885$, des premières meules d'émeri vitrifiées par la Compagnie Norton de Worcester a eu une répercussion considérable sưr l'industrie mécanique, où le travail par meulage tend à remplacer, dans bien des cas, le travail par tournage.

Historique. - Cependant l'émeri et le corindon présentent l'inconvénient d'avoir une composition et par suite une dureté variables, non seulement avec les centres d'extraction, mais encore avec les régions d'un même gisement. Il était par suite impossible d'assurer la fabrication de meules de qualités identiques, à des époques différentes. Le seul remède était la substitution aux abrasifs naturels d'un produit artificiel, de struclure homogène el de composition suffisamment constante.

Les propriétés abrasives remarquables de l'alumine fondue paraissent avoir été découvertes à Froges, en r8go, lors de la fabrication des ferro-aluminiums et cupro-aluminiums par le procédé Héroult, qui consistait à électrolyser, dans un four à cuve, de l'alumine, dont le point de fusion est de $2030^{\circ}$, en présence d'une cathode de fer ou de cuivre liquide. Au moment de la coulée de l'alliage, on laissait échapper une quantité plus ou moins grande d'alumine fondue; on remarqua la grande dureté de ce laitier ct l'on fit quelques essais, en vue de son utilisation comme poudre de polissage. Le produit n'cut pas alors de débouchés suffisants et la fabricalion fut abandonnée.

Quelques annćes plus tard, on songea à la bauxilc ct l'on constala que ce minerai, fondu au four électrique, fournit par refroidisement des bloes à structure cristalline, à base d'alumine anhydre et analogues à l'émeri ou au corindon, selon que la bauxite initiale est plus ou moins riche en impurctés diverses.

On ne tarda pas d'ailleurs à réaliser, à partir de la bauxite impure, unc fusion avec affinage, conduisant à un corindon artificiel relativement pur. La possibilité d'un affinage résulte de ce fait que les impuretés de la bauxite $\left(\mathrm{Fe}^{2} \mathrm{O}^{3}, \mathrm{SiO}^{2}\right.$, $\mathrm{TiO}^{2}$ ) sont plus facilement réduclibles que ne l'est l'alumine, de sorte qu'en trailant au four électrique de la bauxite mélangée à une proportion convenable de charbon, celui-ci met tout d'abord en liberté le fer, le silicium, le titane et enfin un peu d'aluminium. Ces éléments forment un alliage complexe, assez dense, qui se rassemble a a fond $d u$ four et se sépare de l'alumine fondue, que l'on obtiendra d'autant plus pure que la réduction des impuretés aura été plus complète.

Des essais industricls furent entrepris dans celte direction presque simultanément, de 1898 à 1900 , en Bohème el aux Etats-Unis. Ils aboulirent à la fondation des deux premières fabriques de corindon artificiel.

L'une de ces usines est celle de la Bathie, en Savoic; elle appartient à la Société anonyme des Usines de Carborundum et d'Elcetrit réunies. Celte Société poursuivit, dès 1898 , la mise au point de son procédé dans son usine de Benatek, en Bohème. En rgor, Benalck cessa ces essais et l'usine de la Bâthic fut chargéc de la fabrication du produit, qui reçut lo nom commercial d'électrit. La puissance installée est, it la Bâthie, de 3 ooo chevaux, dont 2 ooo pour l'électrit.

La seconde usine est colle du Niagara, aux Ftats-Unis ; elle appartient à la Compagnie Norton, dont nous avons vu lo rôle capital dans l'industrie des meules artificielles. Cette usine, dont la puissance est do 3600 chevaux, a produil en I 912 environ 13 ooo tonnes de corindon synthélique, qu'elle appelle alundum. La Compagnic Norton possède deux autres usines, à Worcester en Amérique et Wesseling en Allemagne, où sont confectionnécs les meules agglomérées à base d'alundum.

En outre de ces deux élablissements, trois autres Socićtés s'occupent du corindon artificjel :

Les Diamantinwerk, qui fabriquent à Rheinfelden l'abrasif alumincux appelé diamantin; La Compagnie française Aloxite, filiale de la Carborundum $\mathrm{C}^{\circ}$, dont l'usine, siluée dans les Hautes-Pyrénés, à Sarrancolin, absorbe environ r ooo chevaux et fabrique un produit nommé aloxile; Ia Société le Boro-Carbone, dont l'usine est au plan d'Arem (Haute-Garonne) et rui consacre une puissance de de 500 à I ooo chevaux à la fusion de la bauxite.

Fabrication. - La bauxile, ayant subi à la mine un premicr triage, suivi de lavage, parvient à l'usine. Là elle est concassée, triée à la main, broyéc et même parfois lavée de nouveau. Elle cst soumise ensuite à une calcination ayant pour but de chasser l'humidité et l'cau de constitution qu'elle renferme.

Cette opération, pour laquelle il scrait peu économique et même incommode de recourir au four électrique, s'ef́ectue généralement dans un four rotalif, incliné légèrenent sur son axe; le chauffage est assuré soil par gazogène, soil par pulvérisation de charbon; le premier moyen est préférable, au point de vue de la pureté du produit calcinć. La bauxile est introduite d'une façon continue à l'extrémité supéricure du four et descend lentement jusqu'à l'orilice inférieur. Une tempéralure de $800^{\circ}$ à $\mathrm{I} 000^{\circ}$ suffit à fournir un produit ne titrant plus que I pour soo d'eau.

La composition de la charge introduite dans te four électrique - bauxite calcinéc, addilionnée ou non de charbon ct d'autres malières - varie suivant les usines el même, pour chaque usine, selon la nature du produit à obtenir. II ne faut pas croire en effet que le corindon pur constitue, malgré sa grande durcté, l'abrașif idéal, répondant à tous les besoins. Dans beaucoup de cas, on cherche à modifier, dans un sens délcrminć, les propriétés abrasives du corindon, en ajoulant au lit de fusion certaines substances. Au nombre de ces additions intentionnelles, on peut citer la magnésié, l'oxyde de fer, la silice, l'oxyde de titane, la glucine et l'aphydride borique.

Dans la fabrication du corindon, comme dans celle du carbure de calcium, on doit distinguer deux modes de fonctionnement du four; la marche par coulce et la marche en 
pain. A chacun de ces modes correspondent des lypes de fours différents.

Dans la marche par coulée, on emploie de préférence des fours à sole conductrice, dans lesquels le courant passe à travers la charge, de l'électrode supérieure mobile à la sole qui constitue la seconde électrode. Ces fours peuvent être fixes et munis d'un trou de coulće placé au niveau de la sole ; lorsque la cuve du four contient une quantité suffisante de produit fondu, on débouche le trou de coulée et le corindon liquide s'écoule dans une lingotière.

Mais le travail par coulée avec un four fixe esl ici défectucux, car le perģage au burin est cxtrêmement pénible et finit même par devenir impossible, étant donnée la grande dureté du produit; d'autre part, le perģage éleclrique ne peut pas être employé, en raison de la trop faible conductibilité du corindon solide.

Il vaut mieux se servir d'un four du type basculant, muni d'un bec supéricur de coulée, analogue en somme au four à acier lléroull; on coule le corindon liquide, en inclinant suffisamment le fortur.

Mais, contraircment à ce qui s'est passé pom le carbure de calcium, la fabrication du corindon par coulée a été peu près délaisscée. Fn effet, Jo refroidissenent brusque, résultant de da coulée, produisait une trempe du corindon qui devenait bcaucoup trop fragile.

Dans la fabricalion par pains, généralement adoptée aújourd'hui, on laisse le four se remplir complètement, en soulevant progressivement les électrodes à mesure que la couche d'alumine fondue angmente d'épaisseur. Lorsque la cuve est pleine, on abandome au refroidissement lent le pain de corindon que l'on extrait ensuite.

Tel est le four de Hall, l'inventcur du procódé de purification électrothermique de la bauxite ; cel appareil, du type à sole conductrice, est muni d'une chambre anmulaire formant récupérateur de chalcur.

La Compagnie Norton, qui empJoya succossivement les fours de Horrey el de Jacobs, adopta en rgoh le four Higgins, spécjalentent étudié en vue de lia fabrication du corindon on pains. Sur une solo réfractitire est posée tune enveloppe en tòle de fer, de forme tronconique, onverte à ses deux bases. On a prévu un dispositif de refroidissement. par ruissellement d'eau sur la paroi extéricure du four. Celui-ci ost du typo à b́lectrodes verticales en sćric, suspendues an-dessus de la chitrge; le comrant arrive par une électrode et sort par l'aulre, apris ayoir formé deux ares avec la matière à traiter.

Il cxisle un type plus recent de four Higgins, à cuve recfangulaire ol double dispositif de ruissellement d'eau.

Le four ì corinton de la Garborunum $\mathrm{C}^{\circ}$ paraît être analogue au précédent; la diflérence essentielle réside dans le foncliomenent du four. Tes deux dectrodes en série plongent ici dans le corindon liquide qui constitue la résistance de chauffage; il n'y aurail pas d'are voltaïque.

Selon le système de four employé, suivant la marche de la fabrication, le rendement de lénergic varie notablement. La production par chcval électriquc-an peut alleindre 3,8 tonnes, s'il y a fusion pure et simple du minerai, tandis qu'elle oscillera aulour de 2,5 à 3 tonnes, dans te cas d'un affinage réducleur ayancé.

Dès qu'un four est plein de corindon, on lève les électrodes ct l'on relire la cure qui est généralement montée sur roues. On la remplace aussitót par une cuve vide, de façon à reprendre la fabrication aussi rapidement que possible et. à diminuer l'usure des élcetrodes par combustion au confact de l'air.
Lorsque le pain est froid, on le sort de la cure et on te nettoie soigneusement. On fait d'abord sauter le disque d'alliage qui forme le culot du pain ; en outre, on enlève, soit à la hachette, soit au burin pneumatique, la croûte périphérique de malièrcs non transformées. On débite ensuite, en blocs plus pelits, à l'aide d'un casse-fonte, la masselotte de corindon dont le poids alteinl parfois 2 à 3 tonnes. Les debris passent alors dans de robustes broyeurs à mâchoires qui les réduisent en une poudre grossière. Le produit subil enfin divers traitements, dont un lavage à l'eau ou à certains agents chimiques. Un tamisage mécanique sépare et classe la matière d’après la grosseur du grain en diverses calégorics de poudres, qui sont transformées en mcules, pierres à aiguiser, toiles à polir, clc.

La fabrication des meules céramiques est extrêmement délicate et nécessite une installation considérable. La Compagnie Norton, qui cst une spécialistc de cette industrie, peut faire jusqu’à I 100 variélés de meules, correspondant toutes à une seule forme et à une même dimension.

Dans les usines où la fusion réductrice de la bauxite, en présence d'une cerlaine quantité de carbone, reste le procédé fondamental ou tout au moins le traitement initial, certaincs précaulions supplémentaircs doivent être prises, en vue de l'obtentión d'un corindon de qualité convenable.

C'est ainsi que l'affinage réducteur a l'jnconvénient de provoquer la formalion d'une petite quantité de carbure d'aluminium qui passe dans l'alumine fondue. Or, ce carbure jouil de la propriété d'être, comme le carbure de calcium, décomposé par l'eau et par l'humidité de l'air; ; le corindon artificiel risquerait done de se déliter et de tomber spontanément en poussière.

Deux types de remèdes sont appliqués. L'un consiste à partir d'une batuxite naturelle ou modifiée, ayant exactement la composilion requise pour l'abrasif, el à opérer unc fusion pure el simple, sans affinage, en s'efforçant même d'éviler la réfuclion par le carbone des électrodes. Ce moyen, préconisé par la Compagnie Norton, s'applique en remplaçant les éleclrodes ordinaires per des électrodes en graphile, moins oxydables et en chargeant bas, à fleur d'arc, de manic̀re à fondre la bauxite aussi rapidement que possible. Mais le produit, même obtenu dans ces conditions, renferme de pelifes quantités de carbure d'aluminium, qu'il faut détruire. Après concassage el broyage, on soumet donc le corindon soit à un grillage oxydant, soit à l'action d'une solution alcaline, soit à un chaulfage à $1200^{\circ}$ avec du chlorure de sodium.

Enfin il ne faul pas oublier que les bloes de corindon conticnnent toujours des grains d'alliage qui restent disséminés dans la masse; on fait subir au produit un triage pour le débarrasser de ces inclusions mélalliques. On effectue de même une séparation électromagnétique des limailles de fer, dont l'introduction cst due à l'usure des broycurs.

Altmine pure. - Mais la fabricalion de corindon el d'émeri arlificicl n'est pas la seule transformation que puisse subir la banxito par voic d'affinage réducleur'. Si l'on ajoute au minerai une quanlité suffisante de charbon et si l'on conduit le four de façon convenable, toutes les impurctés de la bauxite finiront par être éliminées à l'élat de ferroalliage; on oblient ainsi de l'alumine aussi pure que cellc produite par les procédés chimiques couranls et pouvant comme clle être ulilisće à la préparation de l'aluminium.

Cependant la formation de carbure d'aluminium est ici beaucoup plus intense que dans le cas du corindon artificiel, car il faut ajouter à la bauxite un excès notable de charbon; 
aussi l'alumine électrothermique, exposée à l'humidité de l'air, se délite et tombe en poussic̀re, par suite de la décomposition spontanée du carbure d'aluminium.

Depuis la patente anglaise de Hall, qui a fixé le principe de la préparation de l'alumine au four électrique, plusieurs autres brevets ont été pris. D'ailleurs, des essais industriels fort intércssants ont été faits dans diver's pays et la technique du procédé peut être considérée comme établie.

Néanmoins, ce mode de purification de la bauxite n'est pas, croyons-nous, appliqué industriellement. Sa supériorité économique sur les nouveaux procédés chimiques (tels que le procédé à la chaux) est en effet douteuse, d'autant que les alliages obtenus comme sous-produits se conservent mal; la naissance du procédé Serpek est venue d'ailleurs lever toute incertitudè à cet égard.

Enfin l'alumine électrothermique, lor'squ'on l'cmploic pour la préparation de l'aluminium, aurait le grave inconvénient d'encrasser le fond des cuves, soit que le grain d'alumine fondue se dissolve plus difficilement dans le bain de cryolithe que lc grain d'alumine calcinéc, soit que lo carbure d'aluminium qu'elle renferme s'accumule rapidement sur la sole.

Alltages d'alcminuy. - La composition des alliages, que l'on oblient comme sous-produits de la fabrication du corindon et de l'alumine pure, dépend des proportions relatives des impurétés que contient la bauxile. Les constiluants ordinaires de ces alliages sont le fer, le silicium, le tilane et l'aluminium, ce dernier cn quanlité plus ou moins grande, suivant la nature des autres consliluants et les conditions de marche du four.

Avec une bauxite ferrugineuse, pou siliccuse, on aura un ferro-silicium à basse tencur, à r 5 pour ıoo de Si par cxemple. Si l'on part au contraire d'une bauxite blanche siliceuse, on obtiendra un alliage riche en silicium; majs en mème temps une notable quantité d'alumine sera réduite, car il faudra marcher à allure plus chaude et l'on aura finalement un ferro-silico-aluminium, dans lequel la proportion d'aluminium dépassera parfois 5o pour ıoo.

Ces alliages ont le défaut de se désagréger au conlact de l'air, ce qui diminue leur valeur marchande; il cst vraiscmblable que, là encore, il faut chercher l'origine du phénomène dans la présence de carburc d'aluminium.

Plusieurs usines électromélallurgiques fabriquent d'ailleur's spécialement des silico-aluminiums, en réduisant par le charbon la bauxite siliceusc, additionnéc ou non de quartzite. Il n'est plus question ici d'obtenir comme produit principal un laitier de corindon ou d'alumine. La conduite de la fabricalion est alors analogue à celle des ferrosiliciums à haule loneur. L'allure du four est cxicemement chaude : le métal est produit el est accumulé sur la sole à une température bien supérieure à la limite de stabilité du carbure d'aluminium, de sorte que les alliages résistent, sans se déliler, à l'action de l'air.

Ces produits constituent des désoxydants remarquables, fort appréciés par la sidérurgie.

Acuminior ex carbune p'alumatom. - Le four électrique permettanl de préparer, à partir d'une bauxite siliceuse, un alliage d'aluminium el de silicium, on cst évidemment conduit à sc dcmander s'il n'est pas possible de réduire, par le même procédé, l'alumine cxempte d'impuretés el d'obtenir par conséquent do l'aluminium pur.

Ce problème de la préparation électrothermique de laluminium est fort intércssant au point de vic scientifique et même, à certains égards, au point de vue industriel. Il a suscité de nombreux travaux dont les résultats ont été, jusqu'en ces dernières années, peu concordants, mais à la suite desquels certains points fondamentaux peuvent etre considérés comme acquis.

Lorsqu'on cherche à réduire l'alumine par le charbon, lout se passe comme si l'on obtenait, non pas de l'aluminium, mais du cabure d'aluminium, de même qu'en réduisant la chaux par le charbon, on obtient du carbure de calcium et non point du calcium. On prépare aisćment aujourd'hui, par ce procédé, le carbure d'aluminium en blocs compacts, formés de beaux cristaux jaunes.

Si on laisse refroidir lentement ce produit, lors de sa fabrication, ou mieux si on lo maintient à une lempérature de $700^{\circ}$ à $800^{\circ}$, légèrement supérieure au point de fusion de l'aluminium, on voit de l'aluminium métallique sourdre du carbure et l'on a pu recucillir ainsi un poids de métal égal à la moilié de l'aluminium tolal contenu dans le produit; celui-ci prend en outre une coloration de plus en plus foncée, à mesure que le mélal s'ícoule.

L'aluminium mis en liberté dans ces conditions a uno double origine :

$I^{\circ}$ Il provient de la réduclion dirccte de l'alumine par le charbon, de sorte que le produit sortant di four est un melange, en proportions variables, d'aluminium et de carbure d'aluminium, ou plutôt une solution du métal dans le carbure d'aluminium.

$2^{\circ}$ Il provient aussi de la dissociation du carbure d'alumi nium, qu'une élévation de température suffisante décompose cn ses éléments : l'aluminium distille vers les régions froides du four ou se dissout dans le carbure subsistant, tandis que le carbone resie a l'étal de graphite.

On peut, en somms, conclure avec Askenasy que, pour oblenir par voic électrothermique de grandes quantilés d'aluminium, il faudra : on bien ne pas dépasser sensibloment la lempéralure d'ébullition du métal (1 $800^{\circ}$ ), ou bien employer un disposilif de condensation des vapeurs métalliques.

Mais de nouvelles recherches et de nombreux essais scront encore nécessaires, avant que l'on puisse espérer réusail i fixer les bases d'un procédé technique acceplable.

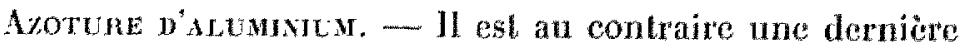
transformation de la bauxite qui est sur le point de prendre" un essor industricl considerable: il s'agit de la fabrication de l'azoture d'aluminium par le procédé Serpek, fabrication pour laquelle deux usines seront incessamment créces, l'unt: en France, près de Gremble, l'autre en Norvòge.

Fn portant dans un four électrique ì une tempiralute d'au moins $1500^{\circ}$ un mélange d'alumine ef de charbon en proportions calculces pour une réduction complete et en envoyant dans le four un courant d'azote, le carbone s'empare de l'oxygene de l'alumine, tandis qus l'azote, se fixant sur l'aluminium mis en liberté, donne de lazoture fou nitrure) d'aluminium. Ce produit, preparé a partiv de l'alumine purc, corme des masses cristallines, a cristaux groupés ct enchevêtrés, de couleur blanche, grise ou blene; il titue en moyenne 30 pour roo d"azole, la teneur théorique étant 34 pour soo.

l.intérêt industriel de l'azoture d'aluminium repose str' la propriété qu'il possède d'être décomposé par l'eau et plus. facilrment encore par les solutions alcalines : l'azote se dom gage à l'éfal de gaz ammoniac, tandis que laluminium so transforme en alumine insoluble ou en aluminate alealin soluble. 
Le procédé Serpek, dont les essais industriels viennent d'être terninés à l'usine de Saint-Jean-de-Maurienne, comporte l'emploi d'un four électrique rotatif, où un mélange de bauxite et de charbon est chauffé dans un courant d'azote. Le produit qui sort du four est de l'azoture à $I_{7-22}$ pour Ioo d'azote ; quant aux impuretés de la bauxite, clles ont été par-tiellement volatilisées, comme le silicium, ou bien elles ont passé à l'état d'alliage complexe disséminé dans la masse.

Cet azolure d'aluminium impur est alors substitué, comme matière premièré, à la bauxite dans les ateliers de fabricalion d'alumine pure travaillant par le procédé Bayer. Mais, au lieu de fournir seulement de l'alumine, ainsi que le fait la bauxite, il donne en même temps du gaz ammoniac que l'on fixe à l'état de sulfate.

I a valeur marchande de ce sel ammoniacal vient donc en déduction importante du prix de revient de l'alumine et, comme l'abaissement du prix de revient de l'aluminium dépend surtout aujourd'hui de la diminution du prix de l'alumine, le procédé Serpck se trouve avoir une importance ca-pitale pour les fabricants d'aluminium.

\section{LES ETUDES GLAGIOLOGIOUES DANS LES ALPES ET LES PYRÉNÉES}

En décrivant l'organisation et le fonclionnement du Service d'élude des Grandes Forces Hydrauliques (Mai rgo5), nous avons indiqué que ce Service comprenait l'étude des glaciers; plus tard, dans notre numéro de Janvier I $\mathbf{9}^{\mathbf{2} 2 \text {, }}$ notre collaborateur, M. Cl. Roux, docteur ès sciences, géologue, donnait le compte rendu des premières publications faites sur les travaux relatifs aux glaciers dans les Alpes. Enfin, ces derniers temps, nous avons, a de nombreuses reprises, analysé les résultals si importants contenus dans les diverses publications du Service.

Ces publications se succèdent maintenant avec régularité; elles deviennent de plus en plus volumincuses, c'est-à-dire de plus en plus bourrées de documents : plans, statistiques, Lableaux, graphiques, ete... et aussi, co qui ne gâte rien, et ce dont il faut féliciter le Service des Faux et Améliorations agricoles qui les fait ćditer, les tomes s'agrémentent de clichés pholographiques loujours plus nombreux et parfois très artistiques. Ils ne sont point insérés dans ces volumes comme des illustrations faites pour distraire l'œil du lecteur, mais à titre documentaire. Et ces pholographies constituent, dans nombre de circonstances, des documents de la plus haute importance.

Tel est notamment, grâce à ce mode d'illustration, le caractère de parfaite documentation qui, dès qu'on le feuillelte, se dégage du Tome III que vient de faire paraître le Service sur les "Etudes glaciologiques (Savoic-Pyrénées)".

Nous ne pouvons pas analyser ce rolume; ses 265 pages grand in $-8^{\circ}$ sont entièrement remplies de rapporls, de notes et de statistiques cxcessivement concises qu'un compte rendu sommaire ne pourrait pas grouper en un article susceptible de donner au lecteur une idée du travail condensé dans ces pages. Aussi croyons-nous préférable de reproduire la table des matières et à titre d'exemples de donner des extraits de quelques chapitres. Et pour que nos Jecteurs se fassent une idée de la riche documentation photographique contenue dans ce volume, nous joignons à ces extraits quelques-uns des clichés qui l'illustrent. Ils nous ont été gracieusement prêtés par les Bureaux du Scrvice des Amélio- rations Agricoles, au Ministère dè l'Agriculture, et nous adressons nos plus sincères remerçements aux. Ingénicurs qui les ont fait mettre à notre disposition.

Le volume comprend deux parties principales: $\mathbf{I}^{\circ}$ Les Etudes glaciologiques en Savoie, par M. Moucrn, inspecteur des Eaux ct Forêts ; $2^{\circ}$ Les Observations glaciaires dans les Pyrénées, par M. Ludovic Gaurier, géologuc.

Nous ferons de plus longs cmprunts à cette deuxième partie, non par ce qu'elle est plus documentée que la première, mais parce qu'elle concerne une région mọins connue de la généralité de nos lecteurs.

Suivant ce que nous venons d'annoncer; voici successivement: la Table des matières du volume, puis des Extraits de quelques chapitres.

\section{TABLE DES MATIETES ET DES PLANCHES}

\section{I. - Études glaciologiques EN SAYOLE}

Texre, - Avant-propos. - Chapitre $1^{\mathrm{er}}$. - Renseignements historiques sur les glaciers : Section $I^{\mathrm{re}}$; Le Massif du Mont-Blanc : §r. Documents généraux, A. Cartes, B. Archives et publications, C. Dessins, gravures, photographies; \$2. Glacier du Tour ; $\$ 3$. Glacier d'Argentière ; \$4. Glacier des Bois ou Mer de Glace, A. Variation de longueur du glacier des Bois, B. Varialion du niveau de la Mer de Glace, C. Vitesse superficielle du Glacier ; $\$ 5$. Le Torrent d'Arveyron; $\$ 6$. Glacier des Bossons; $\S 7$ Glacier de Bionnassay; \$. Glacier de Tré-la-Tète. - Section II : Tarentaise : Glacier de Gébroulaz. - Seclion III : Haute-Maurienne : $\mathrm{I}$. Glacier des Sources de l'Arc; $\$ 2$. Glacier du Mulinet; $\$ 3$. Glacier du Grand Méan ; $\$$ 4. Glacier d'Arnès. - CrrapyTre II : Alimentalion et fusion : $\S \mathrm{I}$. Anciens renseignements sur la neige; $\S 2$. Observations nivométriques: A. Observations faites avant Igoo, Tableaux, B. Observations faites par le Service des Eaux et Forêts ( $a$. Exposé de la méthode ; $b$. Variations des précipitations atmosphériques suivant l'altilude : I. Massif du Monl-Blanc, Ir. Massif de la Belle-Plinier); $\S 3$. La température; $\S 4$. Les précipitations atmosphériques; $\$ 5$. Importance de la fusion : Massif du Mont-Blanc : r. Glacier du Tour, 2. Glacier d'Argentière, 3. Mer de Glace, Glacicr des Bois, 4. Glacier des Bossons, 5. Glacier de Bionnassay. - Recherches sur la périodicilé des crues glaciaires.

Planches. - I. Carte du Nord de la Savoie en 1555 ; Carle du Nord de la Savoie en 1562. - II. Carte des abords du Mont-Blanc en I 742 (dessin de Pierre Martel) ; Carle des environs du Monl-Blanc en $x_{7} 85$, par de Saussure. - III. La vallée de Chamonix vue des moraines des Bossons en $1-7^{3}$ (dessin de Bourril) ; Torrent d'Arveyron, La somrce de l'Arveyron en $\mathrm{I}_{7} 85$ (dessin de Bourrit): - IV. La Mer de Glace vue du Montanvert (dessin de Tackert) ; Vue de la source de l'Arveyron. - V. La vallée de Chamonix vue du Planel (dessin de Hackert, $\mathbf{r}_{7} 87$ ). - VI. Vue perspective de la vallée de Chamonix, du Mont-Blanc et des Montagnes adjacentes dans le Haut-Faucigny on Savoie (publiće en r79o par Chréticn de Méchel). - VII. Vuc de la vallée de Chamonix et du Mont-Blanc (tableau de Linck à la mairie de Chamonix). VIII. Vue de la vallée de Chamonix et du Mont-Blane en rgIt. - IX. Ta source de l'Arveyron en I $_{7} 85$. Vue de l'inléricur (dessin de Bacles' d'Albe) ; La sonrce de l'Arveyron en 18 I 8 (dessin de Bacler d'Albe), - $X$. Torrent de l'Arveyron. La source de l'Arveyion en 1840 (dessin de Lory); Glacier des Bois en r 857 (cliché Buisson, phot. de l'empereur Napoléon III). - XI. Glacier du Tour : Front du glacier (octobre rg10). - XII. Glacier du Tour : Plan, profil de la, ligne supérieure. Variations du front du glacier de 5 58 à rgı r. Variations du niveau du glacier, - XIIT. Glacier d'Ar- 